COMMUNICATIONS IN

ANALYSIS AND GEOMETRY

Volume 12, Number 1, 389-415, 2004

\title{
Local splitting structures on nonpositively curved manifolds and semirigidity in dimension 3
}

\author{
Jianguo CaO $^{1}$, Jeff Cheeger ${ }^{2}$, and Xiaochun Rong ${ }^{3}$
}

Let $M^{n}$ denote a closed Riemannian manifold with nonpositive sectional curvature. Let $X^{n}$ denote a closed smooth manifold which admits an $F$-structure, $\mathcal{F}$. If there exists $f: X^{n} \rightarrow M^{n}$ with nonzero degree, then $M^{n}$ has a local splitting structure $S: 1$ ) The universal covering space with the pull-back metric, has a locally finite covering by closed convex subsets, each of which splits isometrically as a product with nontrivial Euclidean factor. 2) This collection of sets and splittings are invariant under the group of covering transformations. 3) The projection to $M^{n}$ of any flat (i.e. Euclidean slice) of $S$ is a closed immersed submanifold. The structures, $\mathcal{F}, S$, satisfy a consistency condition. If $\mathcal{F}$ is injective, all orbits have dimension $\geq n-2$ and $f$ induces an isomorphism of fundamental groups, then $S$ is abelian i.e. for all $p \in M^{n}$, there is a flat containing all other flats passing through $p$. By $[\mathrm{CCR}], M^{n}$ carries a $C r$-structure which is compatible with $S$. For $n=3$, these conclusions hold even if the extra assumptions on $\mathcal{F}$ are dropped. Moreover, up to isomorphism, the $C r$-structure on $M^{3}$ arising from the construction of $[\mathrm{CCR}]$ is independent of the particular nonpositively curved metric.

\section{Introduction.}

\section{a. Background.}

A classical result due to Heintze and Margulis states that if $M^{n}$ is compact, with $-1 \leq \sec _{M^{n}}<0$, then there exists at least one point at which the injectivity radius is bounded below by a constant, $\epsilon(n)>0$. The HeintzeMargulis theorem is proved by showing that if the conclusion were false,

\footnotetext{
${ }^{1}$ Supported partially by NSF Grant DMS 0102552.

${ }^{2}$ Supported partially by NSF Grant DMS 0104128.

${ }^{3}$ Supported partially by NSF Grant DMS 0203164.
} 
there would exist an abelian subgroup of rank $\geq 2$, thereby contradicting Preismann's theorem, $[\mathrm{Pr}]$.

Buyalo studied the possible failure of the Heintze-Margulis theorem in the context of compact manifolds with nonpositive curvature, $-1 \leq \sec _{M^{n}} \leq 0$; [Bu1-5]. Thus, he investigated the consequences of the assumption that at every point, the injectivity radius is $\leq \epsilon(n)$, for suitable $\epsilon(n)>0$.

For $n=3,4$, Buyalo showed the existence of what we call here an abelian local splitting structure. He conjectured that such a structure should exist in any dimension. This was proved in [CCR].

\section{b. Local splitting structures.}

Let $\pi: \widetilde{M}^{n} \rightarrow M^{n}$ denote the universal covering space of $M^{n}$ and $\Gamma \simeq$ $\pi_{1}\left(M^{n}\right)$, the group of isometric covering transformations.

A local splitting structure on $M^{n}$ is a locally finite collection of closed convex sets, $\left\{Z_{i}\right\}$, and for each $Z_{i}$, an isometric splitting, $Z_{i}=D_{i} \times \mathbb{R}^{k_{i}}$, with $k_{i} \geq 1$, such that:

$$
\bigcup_{i} Z_{i}=\widetilde{M}^{n}
$$

(0.1.2) The collection of sets, $Z_{i}$, and isometric splittings, $D_{i} \times \mathbb{R}^{k_{i}}$, is invariant under the action of $\Gamma$.

(0.1.3) Let $\Gamma_{i}$ denote the abelian subgroup of $\Gamma$ consisting of elements which leave $Z_{i}$ invariant, act by translation on the factor, $\mathbb{R}^{k_{i}}$ and act by the identity on the factor, $D_{i}$. Then $\mathbb{R}^{k_{i}} / \Gamma_{i}$ is compact.

A Euclidean slice $F=w_{i} \times \mathbb{R}^{k_{i}} \subset Z_{i}$, is called a flat. Its projection, $\pi(F)$, to $M^{n}$, is called a projected flat. By $(0.1 .3)$, projected flats are closed immersed submanifolds.

A local splitting structure is called abelian if for all $p \in \widetilde{M}^{n}$, there is maximal flat $F_{p}$ i.e. $F_{p}$ contains all flats that contain $p$; compare [CCR].

\section{c. Nonpositive curvature and local splitting structures.}

For $\gamma \in \Gamma$, the minimum set, $\operatorname{Min}(\gamma)$, is the set of points at which the displacement function, $\delta_{\gamma}(\widetilde{x})=d(\widetilde{x}, \gamma(\widetilde{x}))$, takes its minimum. For any subset, $\mathcal{C} \subset \pi_{1}\left(M^{n}\right)$, we put

$$
\operatorname{Min}(\mathcal{C})=\bigcap_{\gamma \in \mathcal{C}} \operatorname{Min}(\gamma)
$$


If $M^{n}$ has nonpositive sectional curvature, then by [GW], [LY], the set, $\operatorname{Min}(\mathcal{C})$, is totally convex. If $\mathcal{C} \subset \Gamma$ is an abelian group, then $\operatorname{Min}(\mathcal{C})$ is invariant under $\mathcal{C}$. Moreover, there is an isometric splitting,

$$
\operatorname{Min}(\mathcal{C})=D \times \mathbb{R}^{\operatorname{rank}(\mathcal{C})},
$$

such that each $\gamma \in \mathcal{C}$ acts by the identity on the factor $D$ and by translation on the Euclidean factor $\mathbb{R}^{\operatorname{rank}(\mathcal{C})}$. It follows that $\mathbb{R}^{\operatorname{rank}(\mathcal{C})} / \mathcal{C}$ is compact and that $\mathcal{C}$ has finite index in the subgroup, $\Gamma(\mathcal{C})$, of all elements of $\Gamma$ which leave $\operatorname{Min}(\mathcal{C})$ invariant, act by the identity on the factor $D$ and by translation on the Euclidean factor $\mathbb{R}^{\operatorname{rank}(\mathcal{C})}$.

Let $\left\{\mathcal{C}_{i}\right\}$ denote a collection of abelian subgroups of $\Gamma$ which is invariant under conjugation by elements of $\Gamma$. It follows that $\left\{\operatorname{Min}\left(\mathcal{C}_{i}\right)=\right.$ $\left.D_{i} \times \mathbb{R}^{\operatorname{rank}\left(\mathcal{C}_{i}\right)}\right\}$ determines a local splitting structure, $S$, if

$$
\bigcup_{i} \operatorname{Min}\left(\mathcal{C}_{i}\right)=\widetilde{M}^{n}
$$

An abelian local splitting structure is defined by the collection of all nonempty sets (and canonical splittings)

$$
\bigcap_{j} \operatorname{Min}\left(\mathcal{C}_{i_{j}}\right)
$$

provided the following additional condition holds:

(0.2.2) If $\operatorname{Min}\left(\mathcal{C}_{i_{1}}\right) \cap \operatorname{Min}\left(\mathcal{C}_{i_{2}}\right) \neq \emptyset$ then the subgroup generated by $\mathcal{C}_{i_{1}}, \mathcal{C}_{i_{2}}$ is abelian.

\section{d. F-structures.}

An $F$-structure, $\mathcal{F}$, is a topological structure which extends the notion of a torus action on a manifold; see [CG1-2]. For the purposes of this paper, the more significant concept is that of an atlas (of charts) for an $F$-structure. An $F$-structure is an equivalence class of atlases under a natural equivalence relation. This is analogous to the relation between a differentiable structure on a topological manifold and the atlases for that structure.

An atlas for an $F$-structure on a manifold, $X^{n}$, is defined by a collection of triples $\left\{\left(U_{i}, V_{i}, T^{k_{i}}\right)\right\}$, called charts, where $\left\{U_{i}\right\}$ is an open cover of $X^{n}$ and the torus, $T^{k_{i}}$, acts effectively on a finite normal covering, $\pi_{i}: V_{i} \rightarrow U_{i}$, such that the following conditions hold: 
(0.3.1) There is a homomorphism, $\rho_{i}: \Gamma_{i}=\pi_{1}\left(U_{i}\right) \rightarrow \operatorname{Aut}\left(T^{k_{i}}\right)$, such that the action of $T^{k_{i}}$ extends to an action of the semidirect product $T^{k_{i}} \ltimes_{\rho_{i}} \Gamma_{i}$.

(0.3.2) If $U_{i_{1}} \cap U_{i_{2}} \neq \emptyset$, then $U_{i_{1}} \cap U_{i_{2}}$ is connected. If $k_{i_{1}} \leq k_{i_{2}}$, then on a suitable finite covering of $U_{i_{1}} \cap U_{i_{2}}$, there exist lifted actions and an injection, $\ell_{i_{1}, i_{2}}: T^{k_{i_{1}}} \rightarrow T^{k_{i_{2}}}$, such that the action of $T^{k_{i_{1}}}$ is given by the composition of $\ell_{i_{1}, i_{2}}$ with the action of $T^{k_{i_{2}}}$.

The structure, $\mathcal{F}$, is called pure if $k_{i}$ is independent of $i$. It is called polarized if for all $i$, every $T^{k_{i}}$ orbit has dimension $k_{i}$.

We say that $\left\{\left(U_{i}, V_{i}, T^{k_{i}}\right)\right\}$ and $\left\{\left(U_{i}^{\prime}, V_{i}^{\prime}, T^{k_{i}}\right)\right\}$ are equivalent if there is a diffeomorphism, $h: M^{n} \rightarrow M^{n}$, such that $h\left(U_{i}\right)=U_{i}^{\prime}$ and the induced map, $\hat{h}_{i}: V_{i} \rightarrow V_{i}^{\prime}$, induces an equivalence of $T_{k_{i}}$ actions, for all $i$.

The compatibility condition on lifted actions, (0.3.2), implies that $X^{n}$ decomposes as a disjoint union of orbits, $\mathcal{O}$, each of which carries a natural flat affine structure. The orbit containing $x \in X^{n}$ is denoted $\mathcal{O}_{x}$. The dimension of an orbit of minimal dimension is called the rank of the structure.

An $F$-structure is called injective if for each orbit, $\mathcal{O}_{x}$, the inclusion map, $\pi_{1}\left(\mathcal{O}_{x}, x\right) \rightarrow \pi_{1}\left(X^{n}, x\right)$, is an injection.

\section{e. Fundamental groups of orbits and their maximal abelian subgroups.}

Since the orbits have natural flat structures, by the Bieberbach theorem, $\pi_{1}\left(\mathcal{O}_{x}, x\right)$ has a unique maximal normal abelian subgroup. The image of this subgroup in $\pi_{1}\left(X^{n}, \underline{x}\right)$ is denoted $A\left(\mathcal{O}_{x}\right)$.

Fix a base point $\underline{x}$. If $\left\{\left(U_{i_{j}}, V_{i_{j}}, T^{k}\right)\right\}$ is a subcollection of charts (with $k$ independent of $j$ ) for which $\bigcup_{j} U_{i_{j}}$ is connected, then on $\bigcup_{j} U_{i_{j}}$, up to subgroups of finite index and conjugacy in $\pi_{1}\left(X^{n}, \underline{x}\right)$, the group, $A\left(\mathcal{O}_{x}\right)$, is independent of $x \in \bigcup_{j} U_{i_{j}}$. In the application, the ambiguity up to subgroups of finite index will not matter. So from now on, this ambiguity will be suppressed in our notation.

Let $x \in \bigcup_{j} U_{i_{j}}$. Given a homotopy class of curves from $x$ to $\underline{x}$ we get a well defined subgroup $A=A\left(\mathcal{O}_{x}\right) \subset \pi_{1}\left(X^{n}, x\right)$. Let $b_{1} \leq b_{2}$ and let $c_{1}$ : $\left[0, b_{1}\right] \rightarrow X^{n}, c_{2}:\left[0, b_{2}\right] \rightarrow X^{n}$, satisfy $c_{2} \mid\left[0, b_{1}\right]=c_{1}, c_{2}\left(\left[b_{1}, b_{2}\right]\right) \subset \bigcup_{j} U_{i_{j}}$. Then (up to subgroups of finite index) $c_{1}, c_{2}$ determine the same abelian subgroup $A$.

If $\bigcup_{j} U_{i_{j}} \cap U_{\underline{i}} \neq \emptyset$, with $k=k_{i_{j}} \leq k_{\underline{i}}$, as in $(0.3 .2)$, and $c_{2}\left(b_{2}\right) \in U_{i_{2}}$, then the corresponding abelian subgroups satisfy $A \subset A_{\underline{i}}$. 


\section{f. Existence of local splitting structures.}

An $F$-structure, $\mathcal{F}$, on $X^{n}$ and a local splitting structure, $S$, on $M^{n}$ are said to be consistent if there exists $\underline{f}: X^{n} \rightarrow M^{n}$, mapping every orbits of $\mathcal{F}$ into projected flats of $S$, preserving the flat affine structures. Such a map, $\underline{f}$, is called a consistency map. We write $\underline{f} \in C(\mathcal{F}, S)$.

Theorem A. Let $X^{n}, M^{n}$ denote closed manifolds such that $X^{n}$ admits an $F$-structure $\mathcal{F}$. If there exists $f: X^{n} \rightarrow M^{n}$ with nonzero degree, then every metric of nonpositive sectional curvature on $M^{n}$, admits a local splitting structure for which there is a consistency map, $f \in C(\mathcal{F}, S)$, homotopic to $f$.

If $\mathcal{F}$ is pure and $f$ induces an isomorphism of fundamental groups, the local splitting structure constructed in Theorem A is abelian; see Corollary 4.5 .

According to [CG1, 2], the existence of a metric, $g^{\prime}$, with $\left|\sec _{\left(M^{n}, g^{\prime}\right)}\right| \leq 1$, whose injectivity radius is everywhere $\leq \epsilon(n)$, a sufficiently small constant, is equivalent to the existence of an $F$-structure of positive rank on $M^{n}$. Hence, from Theorem A, we get the following strengthened version Heintze-Margulis theorem.

Theorem 0.4 There exists $\epsilon(n)>0$, such that if $M^{n}$ is a closed manifold which admits a metric with nonpositive sectional curvature and negative Ricci curvature at some point, then for every metric with $\left|\sec _{M^{n}}\right| \leq 1$, there is a point at which the injectivity radius is at least $\epsilon(n)$.

\section{g. Rank $n-2$ structures; existence of abelian local splitting structures.}

A polarized $F$-structure is called a graph structure if there exists an atlas, $\mathcal{G}=\left\{\left(U_{i}, V_{i}, T^{k_{1}}\right)\right\}$, for which the following conditions are satisfied:

(0.5.1) Every point in $M^{n}$ is contained in at most two distinct $U_{i}$.

(0.5.2) $\quad k_{i}<n-1$ for some $i$ and $k_{i}=n-1$ for some $i$.

As a consequence of (0.5.2), if $\mathcal{F}$ is a graph structure, then $\mathcal{F}$ is not pure.

If for example, all orbits of a polarized $F$-structure, $\mathcal{F}$, have dimension $\geq n-2$, it is clear that $\mathcal{F}$ is a graph structure.

In general, for any chart, $\left(U_{i}, V_{i}, T^{n-1}\right)$, the set, $U_{i}$, is diffeomorphic to $(-\epsilon, \epsilon) \times \mathcal{O}_{i}$, for some $n-1$ dimensional orbit $\mathcal{O}_{i}$. If $\mathcal{F}$ is a graph structure, then the complement of the union of all such $U_{i}$ is a disjoint union 
of connected submanifolds, $N_{i}$, each of whose boundaries might have several components. Every boundary component of $N_{i}$ is diffeomorphic to some $n-1$ dimensional orbit $\mathcal{O}_{j}$. The restriction of $\mathcal{F}$ to each $N_{i}$ is pure polarized.

Let $U_{2}$ satisfy $k_{2}=n-1$. After fixing a base point and path as in subsection e., we get an abelian subgroup, $A_{2}$, of rank $n-1$, and subgroups $A_{1}, A_{3} \subset A_{2}$, corresponding to those $N_{1}, N_{3}$, which meet the boundary components, $\{-\epsilon\} \times \mathcal{O}_{2},\{\epsilon\} \times \mathcal{O}_{2}$, of $U_{2}$, where possibly, that $N_{1}=N_{3}$.

Theorem B. Let $X^{n}, M^{n}$, denote closed manifolds such that $X^{n}$ admits an injective $F$-structure, $\mathcal{F}$, for which all orbits have dimension $\geq(n-2)$. If there exists $f: X^{n} \rightarrow M^{n}$ with nonzero degree, which induces an isomorphism of fundamental groups, then every metric of nonpositive sectional curvature on $M^{n}$ admits an abelian splitting structure, for which there is a consistency map, $\underline{f} \in C(\mathcal{F}, S)$, homotopic to $f$.

\section{h. Compatibility of abelian local splitting structures and}

\section{$\mathrm{Cr}$-structures.}

An $F$-structure, $\mathcal{F}$, on $X^{n}$ and a local splitting structure, $S$, on $M^{n}$ are called compatible if there is an atlas for $\mathcal{F}$ and a diffeomorphism, $f$, such that for every projected flat, $\pi(F)$, there exists an orbit, $\mathcal{O}$, such that $\underline{f}(\pi(F))=\mathcal{O}$, and $f: \pi(F) \rightarrow \mathcal{O}$ preserves flat affine structures. (Although this condition is slightly stronger than the one given in $[\mathrm{CCR}]$, statements and proofs in [CCR] which concern compatibility remain valid.)

It is not difficult to see that compatibility between $\mathcal{F}$ and $S$ implies consistency. Moreover, a local splitting structure for which there exists a compatible $F$-structure is clearly abelian.

For manifolds, $M^{n}$, of nonpositive curvature, a kind of strengthened converse holds. Any abelian local splitting structure on $M^{n}$ is compatible with an essentially canonical injective $F$-structure on $M^{n}$, for which the relevant atlas satisfies the following conditions (0.6.1), (0.6.2); see [CCR] for the general case and compare [Bu1-5] for dimensions 3,4. An atlas, $\left(U_{i}, V_{i}, T^{k_{i}}\right)$, for an injective $F$-structure which satisfies $(0.6 .1),(0.6 .2)$, is called a $C r$-structure.

(0.6.1) For each $V_{i}$, there is diffeomorphic to a product, $B_{i} \times T^{k_{i}}$, such that the induced action of $T^{k_{i}}$ is by multiplication on the factor, $T^{k_{i}}$, and by the identity on $B_{i}$.

(0.6.2) If $i_{1}, i_{2}$, are distinct, with $U_{i_{1}} \cap U_{i_{2}} \neq \emptyset$, then $k_{i_{1}} \neq k_{i_{2}}$. 


\section{i. Semirigidity in dimension 3 .}

Buyalo showed that in dimension 3, given an arbitrary $C r$-structure on $X^{3}$, a homotopy equivalence, $f: X^{3} \rightarrow M^{3}$ and an arbitrary metric with nonpositive curvature on $M^{3}$, it is possible to modify the $C r$-structure in such a way that it becomes compatible with the given nonpositively curved metric; see [Bu3-5] and compare [Sc]. (He also conjectured that a similar result might hold in higher dimensions; compare Theorem B and see Section 4.) Buyalo did not address the issue of whether or not the modified $C r$-structure on $X^{3}$ actually depends on the particular nonpositively curved metric on $M^{3}$.

According to Theorem $\mathrm{C}$ below, the hypothesis of Buyalo's result can be weakened and the conclusion strengthened. The conclusion of Theorem $\mathrm{C}$ states that there exists a fixed $C r$-structure on $M^{3}$ which is compatible with every metric of nonpositive curvature on $M^{3}$. Moreover, the constructed structure is unique up to isomorphism.

Theorem C. Let $X^{3}$ admit an F-structure, $\mathcal{F}$, and assume that $f: X^{3} \rightarrow$ $M^{3}$ is a homotopy equivalence. Then every metric of nonpositive curvature on $M^{3}$ admits an abelian structure. Moreover, up to isomorphism, this $\mathrm{Cr}$ structure is independent of the particular nonpositively curved metric.

The existence part of Theorem $\mathrm{C}$, requires the construction (in dimension 3 ) of an associated $F$-structure, $\mathcal{F}^{\prime}$, which is either pure or which satisfies the assumptions of Theorem B. For this (using $n=3$ ) it is not necessary to assume that $\mathcal{F}$ is injective. From Theorem B, we obtain an abelian local splitting structure on $M^{3}$, and with the above mentioned result from [CCR], a compatible $\mathrm{Cr}$-structure which is determines a simple graph structure in the sense of Waldhausen. The proof of Theorem $\mathrm{C}$ is completed by appealing to a result of $[\mathrm{Wa1}, 2]$ asserting the uniqueness (up to homeomorphism) of simple graph structures on a 3-manifold whose universal covering space is contractible.

If, as in Theorem $\mathrm{C}, M^{n}$ has the property that the exists a $C r$-structure on $M^{n}$ which is compatible with every nonpositively curved metric, we say that the moduli space of nonpositively curved metrics on $M^{n}$ is semirigid; compare the discussion in [Le], which concerns certain 3 dimensional cases.

It seems possible that semirigidity holds for manifolds satisfying the assumptions of Theorem B; see also Section 4 for more general conjectures.

The rest of the paper is organized as follows:

In Section 1 we prove Theorem A. In Section 2 we prove Theorem B. 
In Section 3 we prove Theorem C.

In Section 4, we discuss the notion of semirigidity.

\section{Proof of Theorem A.}

\section{a. Outline of the proof.}

In this subsection, we outline the proof of Theorem A. Details are provided in the subsections which follow.

Recall that to an atlas, $\left\{\left(U_{i}, V_{i}, T^{k_{i}}\right)\right\}$, we associate the collection $\left\{\left[\Gamma_{i}\right]\right\}$, of conjugacy classes (up to subgroups of finite index) of abelian subgroups of $\pi_{1}\left(X^{n}, \underline{x}\right)$; see Section 0. Let $\left[A_{i}\right]$ denote the conjugacy class of $f_{*}\left(\Gamma_{i}\right)$. Put $S=\left\{\operatorname{Min}\left(A_{i}\right)\right\}$, with $\left.A_{i} \in\left[A_{i}\right] \neq[e]\right\}$. Since $A_{i}$ is abelian, if $A_{i} \neq\{e\}$, then $\operatorname{Min}\left(A_{i}\right)=D_{i} \times \mathbb{R}^{\operatorname{rank}\left(A_{i}\right)}$; see [GW], [LY].

We will construct a map, $\widehat{f}: X^{n} \rightarrow M^{n}$, homotopy to $f$ such that $\widehat{f} \in C(\mathcal{F}, S)$ i.e. $S$ is a local splitting structure and $\widehat{f}$ is a consistency map with respect to $\mathcal{F}$ and $S$.

For every $\left(U_{i}, V_{i}, T^{k_{i}}\right)$ and every orbit, $\mathcal{O}_{i}$, we apply the heat equation for harmonic maps to the map $f\left(\mathcal{O}_{i}\right)$. By letting $t \rightarrow \infty$, the collection of all such maps gives rise to a map, $f_{i, \infty}: U_{i} \rightarrow M^{n}$, homotopic to $f \mid U_{i}$.

Let $\widetilde{\mathcal{O}}_{i}$ denote the lift of $\mathcal{O}_{i}$ to the universal covering space, $\widetilde{X}^{n}$, and let $\widetilde{f}_{i, \infty}$ denote the lift of $f_{i, \infty}$ to $\widetilde{X}^{n}$. Then $\widetilde{f}_{i, \infty}\left(\widetilde{O}_{i}\right) \subseteq \operatorname{Min}\left(A_{i}\right)$.

By means of an equivariant partition of unity, the collection of maps, $\left\{f_{i, \infty}\right\}$, can be glued together to obtain a single map, $\widehat{f}: X^{n} \rightarrow M^{n}$, homotopic to $f$.

For $x \in X^{n}$, let $\left(U_{1}, V_{1}, T^{k_{1}}\right), \ldots,\left(U_{s}, V_{s}, T^{k_{s}}\right)$ denote those charts which contain $x$. We call $x$ a multiple point, if $s>1$. If a principle orbit, $\mathcal{O}_{1}$, is homotopically trivial, or equivalently, $A_{1}=\{e\}$, then $f_{i, \infty}\left(\mathcal{O}_{i}\right)$ has dimension $<k_{i}$ for all orbits $U_{i}$. Hence, $f_{i, \infty}\left(U_{i}\right)$ has dimension $<n$, and thus $\widehat{f}\left(U_{i}\right)$ has dimension $<n$. If $A_{1} \neq\{e\}$, then by the construction of $\widehat{f}$ it follows that $\widehat{f}(x) \in \pi\left(\operatorname{Min}\left(A_{1}\right)\right)$. Because $S$ is locally finite, we conclude

$$
M^{n}=\bigcup_{A_{i} \neq\{e\}} \pi\left(\operatorname{Min}\left(A_{i}\right)\right)
$$

i.e. $S$ is a local splitting structure.

The consistency condition (defined prior to Theorem A) requires that $\widehat{f}\left(\mathcal{O}_{x}\right)$ is a projected flat of $S$; equivalently, for any multiple point $x$, 
$\widehat{f}\left(\mathcal{O}_{x}\right)=\widehat{f}\left(\mathcal{O}_{s}\right)$ is a projected flat of $\operatorname{Min}\left(A_{s}\right)$. Since this may not hold (see Example 1.9) the construction must be slightly adjusted.

We modify the charts of the atlas, $\left\{\left(U_{i}, V_{i}, T^{k_{i}}\right)\right\}$, to obtain an equivalent atlas, $\left\{\left(U_{i}^{\prime}, V_{i}^{\prime}, T^{k_{i}}\right)\right\}$, such that the following property holds: If $x$ is a multiple point of $\left\{\left(U_{i}, V_{i}, T^{k_{i}}\right)\right\}$, then $x$ is not a multiple point of $\left\{\left(U_{i}^{\prime}, V_{i}^{\prime}, T^{k_{i}}\right)\right\}$. Moreover, if $x$ is a multiple point of $\left\{\left(U_{i}^{\prime}, V_{i}^{\prime}, T^{k_{i}}\right)\right\}$, then the orbit in $U_{1}^{\prime}, \mathcal{O}_{1}^{\prime}$, coincides with $\mathcal{O}_{1}=\mathcal{O}_{x}$. It is straightforward to check that if we construct $\widehat{f}$ using $\left\{\left(U_{i}^{\prime}, V_{i}^{\prime}, T^{k_{i}}\right)\right\}$, then $\widehat{f} \in S(\mathcal{F}, S)$. Note that since the new atlas is equivalent to the old one, the associated splitting structure is the same in both cases.

\section{b. Heat flows and harmonic maps.}

The construction of $\widehat{f}$ described in the above uses the heat flows. We briefly recall some relevant facts from $[\mathrm{ES}],[\mathrm{Ha}]$.

Let $N^{m}$ and $M^{n}$ denote closed Riemannian manifolds. A smooth map, $f: N^{m} \rightarrow M^{n}$, is called harmonic if $\Delta f=0$, where $\Delta$ is the Laplacian on maps.

In general, the homotopy class of given a $C^{1}$-map $f$, need not contain any harmonic map. If $M^{n}$ has nonpositive curvature, then there is a harmonic map homotopic to $f$, which minimizes energy in the homotopy class, and which can be obtained by solving the heat equation,

$$
\begin{gathered}
\frac{\partial v(x, t)}{\partial t}=\Delta v(x, t), \quad(x, t) \in N^{m} \times[0, \infty), \\
v(x, 0)=f(x) .
\end{gathered}
$$

Given two maps, $f, h: N^{m} \rightarrow M^{n}$, put $d(f, h)=\sup _{x \in N^{m}} d(f(x), h(x))$. The following result is due to Hartman.

Theorem 1.2 (Har). Let $M^{n}$ denote a closed manifold with nonpositive curvature. For all $t \geq 0$, the heat equation (1.1) has a unique solution, $v(x, t): N^{m} \times[0, \infty) \rightarrow M^{n}$, which satisfies the following conditions:

(1.2.1) The map, $v(x, t)$, is smooth for $t>0$ and $v_{f}(x)=\lim _{t \rightarrow \infty} v(x, t)$ is a harmonic map homotopic to $f$.

(1.2.2) The set of homotopy harmonic maps in a given homotopy class is path-connected. 


\section{(1.2.3) If $h: N^{m} \rightarrow M^{n}$ is another $C^{1}$-map such that $d(f, h)<\operatorname{inj}\left(M^{n}\right) / 2$, then}

$$
d\left(v_{f}(\cdot, t), v_{h}(\cdot, t)\right) \leq d(f, h)
$$

For convenience, we put $u(x, t)=v\left(x, \tan \left(\frac{\pi t}{2}\right)\right)$. Thus, $u(x, 1)=v_{f}(x)$.

\section{c. The construction of $\widehat{f}$.}

Let $f: X^{n} \rightarrow M^{n}$ be as in Theorem A. Without loss of generality, we can assume that $f$ is $C^{1}$. We will deform $f$ to a map $\widehat{f}: X^{n} \rightarrow M^{n}$ which satisfies Theorem A.

Fix an atlas, $\left\{\left(U_{i}, V_{i}, T^{k_{i}}\right)\right\}$, and choose a partition of unity $\left\{\lambda_{i}\right\}$, associated to the open cover $\left\{U_{i}\right\}$, the level sets of whose functions are invariant under the local torus actions. Without loss of generality, we can assume that the charts, $\left\{\left(U_{i}, V_{i}, T^{k_{i}}\right)\right\}$, have been chosen such that if $U_{i} \cap U_{j} \neq \emptyset$, then any orbit in $U_{i}$ is contained in some orbit in $U_{j}$ or vice versa.

Since there is a finite covering space of $U_{i} \cap U_{j}$ on which both $T^{k_{i}}$ and $T^{k_{j}}$ act, we can view $T^{k_{i}} \subseteq T^{k_{j}}$ or vice versa. With this understanding, we fix an invariant metric for the local torus actions; see [CG1].

We now give the construction of the map $\widehat{f}$. The existence of a local splitting structure with which $\widehat{f}$ is consistent will be verified in subsections e-f.

Choose (inductively) invariant metrics on all $\left\{T^{k_{i}}\right\}$ such that if $T^{k_{i}} \subseteq T^{k_{j}}$ then the metric on $T^{k_{i}}$ is the restriction of the $T^{k_{j}}$-metric on $T^{k_{i}}$. Let $T^{k_{i}} \times V_{i}$ be equipped with the product metric. In particular, the restriction of the product metric to each $T^{k}$-factor, $T^{k} \times\{y\}$, is flat.

For each $i$, let $f_{i}: T^{k} \times V_{i} \stackrel{\text { mul }}{\longrightarrow} V_{i} \stackrel{\pi_{i}}{\longrightarrow} U_{i} \stackrel{\left.f\right|_{U_{i}}}{\longrightarrow} M$. For each fixed $y \in V_{i}$, we apply Theorem 1.2 to the heat flow,

$$
\left\{\begin{array}{l}
\frac{\partial u_{i}}{\partial t}=\Delta_{s} u_{i}(s, y, t) \\
u_{i}(s, y, 0)=f_{i}(s, y),
\end{array} \quad(s, y, t) \in T^{k_{i}} \times V_{i} \times[0,1]\right.
$$

and get the solution $u_{i}(\cdot, y, t): T^{k} \times\{y\} \times[0,1] \rightarrow M$.

As $y$ varies, we obtain a family of harmonic maps,

$H_{i}: T^{k_{i}} \times V_{i} \times[0,1] \rightarrow M^{n}, \quad H_{i}(s, y, t)=u_{i}(s, y, t), \quad(s, y, t) \in T^{k_{i}} \times V_{i} \times[0,1]$.

By (1.2.3) the above solution varies continuously with the initial data and thus $H_{i}$ is a continuous map. 
We will construct $\widehat{f}$ by gluing together the maps in the collection, $\left\{u_{i}\right\}$, by means of the center of mass technique.

Let $p \in X^{n}$ and let $p_{i} \in V_{i}$ satisfy $p=\pi_{i}\left(p_{i}\right)$. Let $U_{1}, \ldots, U_{r}$ denote the charts which contain $p$. For each $U_{i}$, let $\alpha_{i}(t)=u_{i}\left(e, p_{i}, t\right)$ denote the trajectory at $q=f(p)$ under the harmonic flow in (1.3).

Let $\pi: \widetilde{M}^{n} \rightarrow M^{n}$ be the Riemannian universal covering map. For each $\widetilde{q} \in \pi^{-1}(q)$ and $t \in[0,1]$, we will define a convex function, $\widetilde{f}_{\widetilde{q}, t}: \widetilde{M}^{n} \rightarrow \mathbb{R}_{+}$, with the property that deck transformations preserve the unique critical point of $\widetilde{f}_{\widetilde{q}, t}$.

Fix any $\widetilde{q} \in \widetilde{M}^{n}$. Lift $\alpha_{i}(t)$ at $\widetilde{q}$ with the terminal point, $\widetilde{q}_{i}(t)$. Clearly, we have:

(1.4) If $\gamma$ is a deck transformation such that if $\widetilde{q}^{\prime}=\gamma(\widetilde{q})$, then $\widetilde{q}_{i}^{\prime}(t)=$ $\gamma\left(\widetilde{q}_{i}(t)\right)$.

We now fix $t$, and thus, $r$ points, $\widetilde{q}_{1}(t), \ldots, \widetilde{q}_{r}(t)$. Using the invariant partition of unity at $p$, we define a function, $f_{\widetilde{q}, t}(\widetilde{y}): \widetilde{M}^{n} \rightarrow \mathbb{R}_{+}$,

$$
\widetilde{f}_{\widetilde{q}, t}(y)=\sum_{i=1}^{r} \lambda_{i}(p)\left[d\left(\widetilde{q}_{i}(t), \widetilde{y}\right)\right]^{2}
$$

Since $\widetilde{M}^{n}$ has nonpositive curvature and since $\lambda_{i}(p) \geq 0$, it follows that $\widetilde{f}_{\widetilde{q}, t}(\widetilde{y})$ is a smooth convex function.

Let $\mathcal{C}_{\widetilde{q}, t} \in \widetilde{M}^{n}$ denote the unique critical point of $\widetilde{f}_{\widetilde{q}, t}$. If $\gamma$ is a deck transformation, by (1.4) we get

$$
\left(\widetilde{f}_{\widetilde{q}, t} \circ \gamma\right)(\widetilde{y})=\widetilde{f}_{\gamma^{-1}(\widetilde{q}), t}(\widetilde{y}) .
$$

Hence, $\gamma\left(\mathcal{C}_{\widetilde{q}, t}\right)=\mathcal{C}_{\gamma^{-1}(\widetilde{q}), t}$ and therefore $\mathcal{C}_{q, t}=\pi\left(\mathcal{C}_{\widetilde{q}, t}\right)$ is independent of the choice of $\widetilde{q} \in \pi^{-1}(q)$.

In view of the above, the map, $H: X^{n} \times[0,1] \rightarrow M^{n}$,

$$
H(p, t)=\mathcal{C}_{f(p), t}, \quad p \in M^{n}, t \in[0,1],
$$

is well defined. Obviously, $H(p, 0)=f(p)$. We put

$$
\widehat{f}(p)=H(p, 1) .
$$

\section{d. Properties of $\widehat{f}$.}

Set $\widehat{f}_{i}(x)=u_{i}\left(e, x_{i}, 1\right): U_{i} \rightarrow M^{n}$, where $x \in U$ and $x_{i} \in V_{i}$ such that $\pi_{i}\left(x_{i}\right)=x$. Then $\widehat{f}$ may be viewed as having been obtained by gluing 
together the members of $\left\{\widehat{f}_{i}\right\}$. In this subsection, we establish some preliminary properties of the maps $\widehat{f}_{i}$. These will be used to show that $\widehat{f}$ satisfies Theorem A.

Given a smooth map, $f: N^{m} \rightarrow M^{n}$, the differential, $f_{*}$, can be regarded as a $T(M)$-valued 1 -form on $M^{n}$. The covariant differential, $\beta(f)=\nabla f_{*}$, is called the second fundamental form of $f$. Note that if $f$ is an immersion, then $\beta(f)$ is indeed the second fundamental form of the immersed submanifold $f\left(N^{m}\right)$. A map $f$ is called totally geodesic if $\beta(f)=0$. In particular, a totally geodesic map sends (closed) geodesics to (closed) geodesics.

We now restrict attention to the situation in which $N^{m}=T^{k}$ is flat and $M^{n}$ is a closed manifold of nonpositive sectional curvature.

Lemma 1.5. Let $T^{k}$ be a flat torus, and let $M^{n}$ be a closed manifold of nonpositive sectional curvature. If $f: T^{k} \rightarrow M$ is a nontrivial harmonic map, then

(1.5.1) $f\left(T^{k}\right)$ is an immersed totally geodesic flat submanifold of dimension $r=\operatorname{rank}\left(f_{*}\left(\pi_{1}\left(T^{k}\right)\right)\right.$.

(1.5.2) A component of $\pi^{-1}\left(f\left(T^{k}\right)\right)$ is contained in a flat of $\operatorname{Min}\left(f_{*}\left(\pi_{1}\left(T^{k}\right)\right)\right.$.

Proof. By [ES] the fact that $M^{n}$ has nonpositive sectional curvature implies that $f: T^{k} \rightarrow M$ is harmonic if and only if $f$ is totally geodesic. Assertions (1.5.1), (1.5.2), are standard consequences of this fact.

Lemma 1.6. Let $\widehat{f}_{i}: U_{i} \rightarrow M^{n}$ be defined as in the above. Then

(1.6.1) The restriction of $\widehat{f}_{i}$ to any orbit in $U_{i}$ is a harmonic map.

(1.6.2) If $\Gamma_{i}=f_{*}\left(\mathcal{C}_{i}\right) \neq 1$, then $\widehat{f}_{i}\left(U_{i}\right) \subseteq \pi\left(\operatorname{Min}\left(\Gamma_{i}\right)\right)$.

Proof. Since the induced metric on any orbit is flat, it suffices to show that the restriction of $\widehat{f}_{i}$ to an orbit is totally geodesic.

Recall that $\widehat{f}_{i}$, has the property that the map, $H_{i}(\cdot, y, 1): T^{k_{i}} \times V_{i} \times$ $\{1\} \rightarrow V_{i} \stackrel{\pi_{i}}{\longrightarrow} U_{i} \stackrel{\widehat{f}_{i}}{\longrightarrow} M^{n}$, obtained from the heat flow (1.4), is harmonic and therefore totally geodesic. Let $T^{k_{y}}$ denote the isotropy group at $y$. Then $p_{s}: T^{s}=T^{k} / T_{y}^{k} \rightarrow \mathcal{O}_{p(y)}$ is a Riemannian submersion and thus totally geodesic. Then, $\widehat{f}_{i}: \mathcal{O}_{p(y)} \rightarrow M^{n}$ satisfies that $\widehat{f}_{i}(x)=H_{i}(x, 1)(t)$ for any $t \in T^{s}$ such that $p_{s}(t)=x$. This shows that $\widehat{f_{i}}$ is totally geodesic. This shows (1.6.1) 
To see (1.6.2), note that $U_{i}$ is the union of $T^{k_{i}}$-orbits. By Lemma 1.5, we see that when restricted to each orbit $\mathcal{O}_{x}$ in $U_{i}$, we have $\widehat{f}_{i}\left(\mathcal{O}_{x}\right) \subseteq \pi\left(\operatorname{Min}\left(\Gamma_{i}\right)\right)$.

\section{e. Existence of the local splitting structure and consistency.}

Recall that to an atlas, $\left\{\left(U_{i}, V_{i}, T^{k_{i}}\right)\right\}$, we associate the collection $\left\{\left[\Gamma_{i}\right]\right\}$, of conjugacy classes (up to subgroups of finite index) of abelian subgroups of $\pi_{1}\left(X^{n}, \underline{x}\right)$. By passing to a subgroup of each $\Gamma_{i}$ with finite index if necessary, we may assume that $\left\{\left[\Gamma_{i}\right]\right\}$ is chosen so that the following condition is satisfied; see the proof of Proposition 1.8.

(1.7) For $x \in X^{n}$, let $\left(U_{1}, V_{1}, T^{k_{1}}\right), \ldots,\left(U_{s}, V_{s}, T^{k_{s}}\right)$ be the charts containing $x, k_{1} \leq \cdots \leq k_{s}$. Then $\Gamma_{i} \subseteq \cdots \subseteq \Gamma_{s}$.

Let $S=\left\{\operatorname{Min}\left(A_{i}\right), A_{i} \in\left[A_{i}\right] \neq[e]\right\}$, where $\left[A_{i}\right]$ denotes the conjugacy class of $f_{*}\left(\Gamma_{i}\right)$.

The following gives a sufficient condition for $S$ to be a local splitting structure with $\widehat{f} \in C(\mathcal{F}, S)$ i.e $\widehat{f}$ is a consistency map.

Proposition 1.8. Given an atlas, $\left\{\left(U_{i}, V_{i}, T^{k_{i}}\right)\right\}$, and an invariant partition of unity $\left\{\lambda_{i}\right\}$, there is a map, $\widehat{f}: X^{n} \rightarrow M^{n}$, satisfying the following conditions:

(1.8.1) If $x \in X^{n}$ is such that $\mathcal{O}_{x}$ is not homotopically trivial, then $\widehat{f}\left(\mathcal{O}_{x}\right)$ is contained in a projected flat of $S$.

(1.8.2) Let $U$ denote the union of orbits which are homotopically trivial. Then $\widehat{f}$ maps each orbit in $U$ to a point and thus $\operatorname{dim}(\widehat{f}(U))<n$.

Proof of local splitting and consistency assuming Proposition 1.8.

We must show

$$
\widetilde{M}^{n}=\bigcup_{A_{i} \neq\{1\}} \operatorname{Min}\left(A_{i}\right) .
$$

Equivalently, $S$ is a local splitting structure. Then (1.8.1) and (1.8.2) imply $f \in C(\mathcal{F}, S)$.

Because the collection of closed subsets, $\left\{\pi\left(\operatorname{Min}\left(A_{i}\right)\right), A_{i} \neq\{1\}\right\}$, is locally finite, the subset,

$$
M^{n} \backslash \bigcup_{A_{i} \neq\{1\}} \pi\left(\operatorname{Min}\left(A_{i}\right)\right)
$$


if not empty, must be open. Since $\widehat{f}$ is an onto map satisfying (1.8.2), this gives a contradiction.

The following example shows that if as in subsection c, we construct $\widehat{f}$ in Proposition 1.8 using the atlas $\left\{\left(U_{i}, V_{i}, T^{k_{i}}\right)\right\}$, then $\widehat{f}$ may not satisfy (1.8.1).

Example 1.9. Consider a mixed $T$-structure on a flat torus $T^{3}=T^{2} \times T^{1}$, with two adjacent charts, one with rank one and the other with rank two. The union of these charts is $T^{2} \times(0,1) \subset T^{2} \times T^{1}$. Assume that the $T^{2}$-orbits in the rank two chart are not totally geodesic submanifolds while $T^{1}$-orbits in the rank one chart are totally geodesic. Thus, in the overlap, each $T^{2}$-orbit is a union of totally geodesic circles.

Let $\mathcal{O}$ denote a $T^{2}$-orbit lying in the overlap of the two charts. The heat flow on the rank two chart deforms the $T^{2}$-orbit to a totally geodesic torus. On the chart of rank one the heat flow acts trivially. Thus, it leaves the $T^{2}$-orbit unchanged.

It follows that $\widehat{f}$ maps the above $T^{2}$-orbit to the gluing via the center of mass construction of the $T^{2}$-orbit and the corresponding totally geodesic 2 -torus arising from the heat flow on the rank two chart. Obviously, $\widehat{f}$ is not a consistency map, since it maps the $T^{2}$-orbit to a torus which is not totally geodesic.

Note that $\widehat{f}$ maps any $T^{1}$-orbit in the rank one chart to a closed geodesic. This is because the images of this orbit under both heat flows are totally geodesic circles. Therefore, their center of mass is a totally geodesic circle as well.

Now suppose that one slightly fattens the rank two chart and shrinks the rank one chart so that the $T^{2}$-orbits on the overlap with respect to the old charts are not in the overlap of the new charts. Then the gluing map constructed using the new charts, maps the $T^{2}$-orbits in the overlap of the old charts to projected flats i.e. the gluing construction with respect to the new charts is consistent (with respect to orbits of the old charts). Indeed, constructing $\widehat{f}$ with a refined atlas, $\left\{\left(U_{i}^{\prime}, V_{i}^{\prime}, T^{k_{i}}\right)\right\}$, as below, is all that is needed for $\widehat{f}$ to satisfy (1.8.1) and (1.8.2).

Lemma 1.10. Given any atlas, $\left\{\left(U_{i}, V_{i}, T^{k_{i}}\right)\right\}$, there is an equivalent atlas, $\left\{\left(U_{i}^{\prime}, V_{i}^{\prime}, T^{k_{i}}\right)\right\}$, such that:

(1.10.1) $\Gamma_{i}=\Gamma_{i}^{\prime}$, for all $i$.

(1.10.2) For $x \in M$, let $U_{1}, \ldots, U_{s}$ denote those $U_{i}$ that contain $x$, where 


$$
k_{1}<\cdots<k_{s} \text {. Then }
$$

$$
x \in U_{s}^{\prime} \backslash \bigcup_{k_{j}<k_{i}} U_{j}^{\prime} .
$$

(1.10.3) For $x \in M$, let $U_{1}^{\prime}, \ldots, U_{s}^{\prime}$ denotes those $U_{i}^{\prime}$ that contain $x$. Then $\mathcal{O}_{x}=\mathcal{O}_{x, 1}^{\prime}\left(\right.$ the $T^{k_{1}}$-orbit in $\left.U_{1}^{\prime}\right)$.

Proof. For each $U_{i}$, let $\widehat{U}_{i}$ denote a small tubular neighborhood of $U_{i}$ such that the pure structure on $U_{i}$ extends to $\widehat{U}_{i}$, and $\left\{\left(\widehat{U}_{i}, \widehat{V}_{i}, T^{k_{i}}\right)\right\}$ is an equivalent atlas.

Then it is easy to see that the collection of charts corresponding to the following sets, $U_{i}^{\prime}$, has the desired properties:

$$
U_{i}^{\prime}=\widehat{U}_{i} \backslash \bigcup_{k_{\ell}>k_{i}} \overline{U_{\ell}}
$$

For $x \in M$, let $\left(U_{1}, V_{1}, T^{k_{1}}\right), \ldots,\left(U_{s}, V_{s}, T^{k_{s}}\right)$, where $k_{1} \leq \cdots \leq k_{s}$, denote those charts such that $x \in U_{i}$. Let $\mathcal{O}_{i}$ denote the $T^{k_{i}}$-orbit in $U_{i}$. Thus, $\mathcal{O}_{1} \subseteq \cdots \subseteq \mathcal{O}_{s}$. Then $\mathcal{O}_{x}=\mathcal{O}_{s}$.

The following lemma implies that $\widehat{f}\left(\mathcal{O}_{1}\right)$ is always a projected flat of $A_{1}$. Note that according to Example 1.9, $\widehat{f}\left(\mathcal{O}_{x}\right)$ may not have this property.

Lemma 1.11. Let $\widetilde{M}^{n}$ denote a complete manifold of nonpositive sectional curvature. Let $C: \triangle^{s} \times \mathbb{R}^{k} \rightarrow D \times \mathbb{R}^{k} \subseteq \widetilde{M}^{n}$ denote a smooth map, with $D \times \mathbb{R}^{k}$ is a closed totally convex subset. Then for fixed $e_{\lambda} \in \triangle^{s}$, with barycentric coordinates, $\left(\lambda_{1}, \ldots, \lambda_{k}\right)$, the image, $C\left(e_{\lambda}, \mathbb{R}^{k}\right)$, is contained in a flat of $D \times \mathbb{R}^{k}$, provided the following conditions hold:

(1.11.1) For $e_{i} \in \triangle$ a vertex (where $1 \leq i \leq s$ ), the map,

$$
C\left(e_{i}, x\right): e_{i} \times \mathbb{R}^{k} \rightarrow y_{i} \times \mathbb{R}^{k} \subset \widetilde{M}^{n}
$$

is a totally geodesic embedding.

(1.11.2) $C\left(e_{\lambda}, x\right)$ is the weighted center of mass, $\left\{C\left(e_{i}, x\right), \lambda\right\}$.

Proof. First, since $f_{i}(x) \in D \times \mathbb{R}^{k}$ which is a convex subset, by (1.11.2) we see that $C\left(e_{\lambda}, x\right) \in D \times \mathbb{R}^{k}$ for all $C\left(e_{\lambda}, x\right)$. It remains to check that $C\left(e_{\lambda}, x\right) \in *_{\lambda} \times \mathbb{R}^{k}$. 
Since $D \times \mathbb{R}^{k}$ is a metric product, for all $(y, z),\left(y^{\prime}, z^{\prime}\right) \in D \times \mathbb{R}^{k}$,

$$
d\left((y, z),\left(y, z^{\prime}\right)\right)^{2}=d\left(y, y^{\prime}\right)^{2}+d\left(z, z^{\prime}\right)^{2} .
$$

Note that $C\left(e_{\lambda}, x\right)$ is the unique critical point of the energy function,

$$
\begin{aligned}
E\left(e_{\lambda}, x\right) & =\sum_{i=1}^{s} \lambda_{i} d\left((y, z),\left(y_{i}, z_{i}\right)\right)^{2} \\
& =\sum_{i=1}^{s} \lambda_{i}\left[d\left(y, y_{i}\right)^{2}+d\left(z, z_{i}\right)^{2}\right] \\
& =\sum_{i=1}^{s} \lambda_{i} d\left(y, y_{i}\right)^{2}+\lambda_{i} \sum_{i=1}^{s} d\left(z, z_{i}\right)^{2} \\
& =E(y)+E(z),
\end{aligned}
$$

where $C\left(e_{\lambda}, x\right)=(y, z), C\left(e_{i}, 0\right)=\left(y_{i}, z_{i}\right)$ and $e_{\lambda}=\left(\lambda_{1}, \ldots, \lambda_{s}\right)$. The above implies that

$$
C\left(e_{\lambda}, x\right)=\left(C\left(\left\{y_{i}\right\}, \lambda\right), C\left(\left\{z_{i}\right\}, \lambda\right)\right),
$$

where $C\left(\left\{y_{i}\right\}, \lambda\right)$ denotes the center of mass of $\left\{y_{i}\right\}$ with weight $\lambda$. The claim follows.

Proof of Proposition 1.8. Given an atlas, $\left\{\left(U_{i}, V_{i}, T^{k_{i}}\right)\right\}$, let $\left\{\left(U_{i}^{\prime}, V_{i}^{\prime}, T^{k_{i}}\right)\right\}$ denote an equivalent atlas as in Lemma 1.10. Using the new atlas and the construction in subsection c, we obtain a map, $\widehat{f}: X^{n} \rightarrow M^{n}$, homotopic to $f$. It remains to check (1.8.1) and (1.8.2) for $\widehat{f}$.

We first verify (1.8.1). If $x \in X^{n}$ is a multiple point of $\left\{\left(U_{i}, V_{i}, T^{k_{i}}\right)\right\}$, then by (1.10.2) $x$ is not a multiple of $\left\{\left(U_{i}^{\prime}, V_{i}^{\prime}, T^{k_{i}}\right)\right\}$. If $x \in U_{i}^{\prime}$, then $\mathcal{O}_{x}=\mathcal{O}_{x}^{\prime}$, and thus $\widehat{f}\left(\mathcal{O}_{x}\right)=\widehat{f}\left(\mathcal{O}_{x}^{\prime}\right)$ is a projected flat of $A_{i}$; see Lemma 1.5.

If $x$ is not a multiple point of $\left\{\left(U_{i}, V_{i}, T^{k_{i}}\right)\right\}$. We can assume that $x$ is a multiple point of $\left\{\left(U_{i}^{\prime}, V_{i}^{\prime}, T^{k_{i}}\right)\right\}$. Let $\left(U_{1}^{\prime}, V_{1}^{\prime}, T^{k_{1}}\right), \ldots,\left(U_{s}^{\prime}, V_{s}^{\prime}, T^{k_{s}}\right)$ denote the charts containing $x$. We will show that (1.10.3) implies that $\widehat{f}\left(\mathcal{O}_{x}\right)$ is a projected flat of $A_{1}$.

Fix $\widetilde{x} \in \widetilde{M}$ with $\pi(\widetilde{x})=\widehat{f}(x)$. Let $\widetilde{y}_{i}=\tilde{\gamma}_{i}(1)$, where $\tilde{\gamma}_{i}(t)$ is the lift at $\widetilde{x}$ of the trajectory, $\gamma_{i}(t)$, of the heat flow in $U_{i}^{\prime}$ (see subsection c). Since by (1.7), we have $\Gamma_{1} \subseteq \cdots \subseteq \Gamma_{s}$, it follows that $\tilde{y}_{i} \in \operatorname{Min}\left(A_{i}\right) \subseteq \operatorname{Min}\left(A_{1}\right)$. Thus, the weighted center of mass, $\widetilde{y}$, of $\left\{\widetilde{y}_{i}\right\}$, associated to the partition of unity, is also in $\operatorname{Min}\left(A_{1}\right)$. By (1.10.3) and the invariance of the partition of unity, we conclude from Lemma 1.11 that $\widehat{f}\left(\mathcal{O}_{x}\right)=\widehat{f}\left(\mathcal{O}_{1}^{\prime}\right)$ is the projected flat of $A_{1}$. 
We now verify (1.8.2). Let $x \in X^{n}$ such that $\mathcal{O}_{x}$ is homotopically trivial. As in the verification of (1.8.1), we consider two distinct cases, depending on whether or not $x$ is a multiple point of $\left\{\left(V_{i}, U_{i}, T^{k_{i}}\right)\right\}$.

Suppose $x$ is a multiple point and let $\left(U_{1}, V_{1}, T^{k_{1}}\right), \ldots,\left(U_{s}, V_{s}, T^{k_{s}}\right)$ denote those charts such that $x \in U_{i}$. By (1.10.2), we have $\mathcal{O}_{x}=\mathcal{O}_{s}^{\prime}$. Thus, by Lemma 1.5 we conclude that $\widehat{f}\left(\mathcal{O}_{s}\right)=\widehat{f}\left(\mathcal{O}_{s}^{\prime}\right)$ is a point.

Suppose $x$ is not a multiple point of $\left\{\left(U_{i}, V_{i}, T^{k_{i}}\right)\right\}$. It suffices to assume that the charts with $x \in U_{i}$ are $\left(U_{1}^{\prime}, V_{1},{ }^{\prime} T^{k_{1}}\right), \ldots,\left(V_{s}^{\prime}, U_{s}^{\prime}, T^{k_{s}}\right)$. By (1.10.3), $\widehat{f}\left(\mathcal{O}_{x}\right)=\widehat{f}\left(\mathcal{O}_{1}^{\prime}\right)$. Since $\widehat{f}\left(\mathcal{O}_{1}^{\prime}\right)$ is obtained from the gluing of $\widehat{f}_{1}\left(\mathcal{O}_{1}^{\prime}\right), \ldots, \widehat{f}_{s}\left(\mathcal{O}_{1}^{\prime}\right)$ (see subsection c), and since each $\widehat{f}_{i}\left(\mathcal{O}_{1}^{\prime}\right)$ is a point (see Lemma 1.5), it follows that $\widehat{f}\left(\mathcal{O}_{1}^{\prime}\right)$ is a point.

\section{Proof of Theorem B.}

\section{a. Alternating sequences.}

Let the collection of groups, $\left\{\Gamma_{i}\right\}$, be defined as in Section 1. Since $f_{*}$ : $\pi_{1}\left(X^{n}\right) \rightarrow \pi_{1}\left(M^{n}\right)$ is an isomorphism, the images under $f_{*}$ of conjugacy classes, $\left[A_{i}\right]$, in $\pi_{1}\left(X^{n}\right)$, are conjugacy classes in $\pi_{1}\left(M^{n}\right)$.

By Theorem A, the sets, $\operatorname{Min}\left(\Gamma_{i}\right)$, together with their canonical splittings, $D_{i} \times \mathbb{R}^{k_{i}}$, satisfy (0.2.1), and hence, determine a local splitting structure $S$.

Note that each component of the union, $Y$, of all $(n-1)$-dimensional orbits is diffeomorphic to $I \times \mathbb{R}^{n-1}$, for some interval $I$. A component of $M^{n} \backslash Y$ will be denoted by $N_{i}$.

In what follows we continue to identify two subgroups if they have a common subgroup of finite index.

A finite sequence, $\left\{\Gamma_{i_{j}}\right\}$, whose first element is either $\Gamma_{i_{1}}$ or $\Gamma_{i_{2}}$ will be called alternating if the following conditions hold:

$$
\begin{aligned}
& \operatorname{rank}\left(\Gamma_{i_{2 j}}\right)=n-1, \text { for all } j \\
& \operatorname{rank}\left(\Gamma_{i_{2 j-1}}\right)=n-2, \text { for all } j \\
& \Gamma_{i_{j_{1}}} \neq \Gamma_{i_{j_{2}}}, \text { if } j_{1} \neq j_{2}
\end{aligned}
$$

The proof of Theorem B is an immediate consequence of the following two assertions.

(2.2.1) If $\Gamma_{i_{k}}, \Gamma_{i_{\ell}}$, are members of an alternating sequence, and $\operatorname{Min}\left(\Gamma_{i_{k}}\right) \cap$ $\operatorname{Min}\left(\Gamma_{i_{\ell}}\right) \neq \emptyset$, then $k, \ell \in\{2 j-1,2 j, 2 j+1\}$, for some $j$. 
(2.2.2) For all $\Gamma_{\alpha}, \Gamma_{\beta}$, there exists an alternating sequence with first member $\Gamma_{\alpha}$ and last member $\Gamma_{\beta}$.

To see (2.2.2), let $\Gamma_{\alpha}, \Gamma_{\beta}$ be given by, $f_{*}\left(A_{\alpha}\right), f_{*}\left(A_{\beta}\right)$, where $A_{\alpha}, A_{\beta}$, are the maximal normal Abelian subgroups of $\mathcal{O}_{\alpha}, \mathcal{O}_{\beta}$. By connecting $\mathcal{O}_{\alpha}$ to $\mathcal{O}_{\beta}$ by a suitable curve, $c$, which meets the boundaries of all $N_{i}$ transversally, we obtain a finite sequence, $A_{i_{j}}$, for which the corresponding sequence, $\left\{\Gamma_{i_{j}}\right\}$, begins with $\Gamma_{\alpha}$, terminates in $\Gamma_{\beta}$ and satisfies (2.1.1), (2.1.2).

A suitably reindexed subsequence satisfies (2.1.3) as well. To see this, if $j(1)$ is the largest subindex such that $\Gamma_{i_{j(1)}}$ is isomorphic to $\Gamma_{i_{1}}$ (always up to subgroups of finite index) we remove the part of the sequence prior to $\Gamma_{i_{j(1)}}$ and renumber so that $\Gamma_{i_{j(1)}}$ becomes $\Gamma_{i_{1}}$. Let $j(2)$ be the largest index of the renumbered sequence such that $\Gamma_{i_{j(2)}}$ is isomorphic to $\Gamma_{i_{2}}$. Then we remove the portion, $\Gamma_{i_{2}}, \ldots \Gamma_{i_{j(2)}-1}$, and renumber such that $\Gamma_{i_{j(2)}}$ becomes $\Gamma_{2}$. By proceeding in this way, after finitely many steps, we obtain the required subsequence. This gives (2.2.2).

Note that two rank $n-2$ abelian subgroups of a rank $n-1$ abelian group either coincide or generate the entire group. Hence, from (2.1.1)-(2.1.3), we get the following additional property.

The subgroup generated by $\Gamma_{i_{2 j-1}}, \Gamma_{i_{2 j+1}}$ is $\Gamma_{i_{2 j}}$.

\section{b. Proof of (2.2.1).}

Fix $j$ such that $\Gamma_{i_{2 j-1}} \neq \emptyset, \Gamma_{i_{2 j+2}} \neq \emptyset$.

It follows from (2.1.1), $\operatorname{Min}\left(\Gamma_{i_{2 j}}\right)$ is isometric to $I \times \mathbb{R}^{n-1}$, for some interval, $I$, which might, apriori, be infinite or semi-infinite. If nonempty, $\partial\left(I \times \mathbb{R}^{n-1}\right)$, is the disjoint union of a pair of totally geodesic submanifolds isometric to $\mathbb{R}^{n-1}$, or just one such. The same holds for $\operatorname{Min}\left(\Gamma_{i_{2 j+2}}\right)$.

By (2.3.1), we have $\operatorname{Min}\left(\Gamma_{i_{2 j-1}}\right) \cap \operatorname{Min}\left(\Gamma_{i_{2 j+1}}\right)=\operatorname{Min}\left(\Gamma_{i_{2 j}}\right)$.

By (2.1.3), $\operatorname{Min}\left(\Gamma_{i_{2 j-2}}\right), \operatorname{Min}\left(\Gamma_{i_{2 j}}\right)$ intersect transversally, or not at all. In particular, $\operatorname{Min}\left(\Gamma_{i_{2 j-2}}\right)$, is not a subset of $\operatorname{Min}\left(\Gamma_{i_{2 j}}\right)$. It follows that if we put $I=[a, b]$, then say $-\infty<a$ and $a \times \mathbb{R}^{n-1} \subset \partial\left(\operatorname{Min}\left(\Gamma_{i_{2 j+1}}\right)\right)$.

Similarly, $b<\infty, b \times \mathbb{R}^{n-1} \subset \partial\left(\operatorname{Min}\left(\Gamma_{i_{2 j-1}}\right)\right)$, and $\operatorname{Min}\left(\Gamma_{i_{2 j-1}}\right) \backslash \operatorname{Min}\left(\Gamma_{2 i}\right)$, $\operatorname{Min}\left(\Gamma_{2 i+1}\right) \backslash \operatorname{Min}\left(\Gamma_{i_{2 j}}\right)$, lie in different components of $\widetilde{M}^{n} \backslash \operatorname{Min}\left(\Gamma_{i_{2 j}}\right)$.

Now it follows that $\operatorname{Min}\left(\Gamma_{i_{2 j-2}}\right), \operatorname{Min}\left(\Gamma_{i_{2 j}}\right)$ cannot intersect transversally. Otherwise, $\operatorname{Min}\left(\Gamma_{i_{2 j-2}}\right) \cap \partial\left(\operatorname{Min}\left(\Gamma_{i_{2 j}}\right)\right)$ would not be contained in $\operatorname{Min}\left(\Gamma_{i_{2 j+1}}\right)$.

As above, $\operatorname{Min}\left(\Gamma_{i_{2 j-3}}\right) \backslash \operatorname{Min}\left(\Gamma_{i_{2 j-2}}\right), \operatorname{Min}\left(\Gamma_{i_{2 j-1}}\right) \backslash \operatorname{Min}\left(\Gamma_{i_{2 j-2}}\right)$, lie in different components of $\widetilde{M}^{n} \backslash \operatorname{Min}\left(\Gamma_{i_{2 j-2}}\right)$. 
Hence, $\operatorname{Min}\left(\Gamma_{i_{2 j-3}}\right) \cap \operatorname{Min}\left(\Gamma_{i_{2 j-1}}\right)=\emptyset$. By induction, it follows that for all $k \leq 2 j-2, \ell \geq 2 j+2, \operatorname{Min}\left(\Gamma_{i_{k}}\right), \operatorname{Min}\left(\Gamma_{i_{\ell}}\right)$ lie in different components of $\widetilde{M}^{n} \backslash \operatorname{Min}\left(\Gamma_{i_{2 j}}\right)$. This gives (2.2.1).

\section{Proof of Theorem C.}

The references for this section are [Ro], [Wa1,2]. The main effort is devoted to establishing the following topological result.

Theorem 3.1. Let $M^{3}$ be a closed 3-manifold whose universal covering space is contractible. If $M^{3}$ admits a nontrivial F-structure, then $M^{3}$ admits an injective F-structure.

First, assuming Theorem 3.1, we prove Theorem C.

Proof of Theorem $C$. Note that the uniqueness statement holds if $M^{3}$ admits a flat metric. Hence, we can assume that $M^{3}$ does not admit any flat metric. By Theorem 3.1 together with Theorem B, every nonpositively curved metric, $g$, on $M^{n}$, admits an abelian local splitting structure.

Note that the uniqueness statement holds if $M^{3}$ admits a flat metric.

Hence, we can assume that $M^{3}$ does not admit any flat metric. Let $\mathcal{C} r(g)$ denote the associated canonical $C r$-structure. We claim that $\mathcal{C} r(g)$ determines a simple graph structure in the sense of Waldhausen, [Wa1,2]; see also [Ro]. Following the discussion in Section 5 of [Ro], we can apply [Wa1,2], to conclude that the isomorphism class of $\mathcal{C} r(g)$ is independent of $g$.

In the proof of Theorem 3.1, following [Ro], we will give a reduction process which leads to our being able to replace a non-trivial $F$-structure by an irreducible structure. We show that if this irreducible $F$-structure were not injective, then the universal covering would not be contractible; a contradiction.

Before beginning the actual argument, we consider a typical example.

Example 3.2. An $F$-structure on $X^{n}$ is called semi-injective if for every orbit, $\mathcal{O}$, the kernel of natural homomorphism, $\pi_{1}(\mathcal{O}) \rightarrow \pi_{1}\left(X^{n}\right)$, is properly contained in $\pi_{1}(\mathcal{O})$.

First, we construct a 3-manifold, $M^{3}$, with infinite fundamental group, which supports a $T$-structure that is polarized but not semi-injective. Then we show that the universal covering, $\widetilde{M}^{3}$, of $M^{3}$, is contractible. 
Let $\Sigma^{2}$ denote a compact surface with non-empty boundary. Let $X^{3}$ denote the result of gluing a solid torus, $D^{2} \times S^{1}$, to $\Sigma^{2} \times S^{1}$, in such a way that $\partial D^{2}$ is identified with the $S^{1}$-factor in $\Sigma^{2} \times S^{1}$. Hence, the homotopy class of the $S^{1}$-factor is trivial in $X^{3}$. Let $M^{3}$ denote a closed 3 -manifold obtained by gluing $X^{3}$ to some compact 3 -manifold, $Y^{3}$, with torus boundary, where $Y^{3}$ is chosen to admit a polarized $T$-structure.

The manifold, $M^{3}$, carries the mixed polarized $T$-structure generated by the $S^{1}$-rotations on $D^{2} \times S^{1}$ and $\Sigma^{2} \times S^{1}$ and the $T$-structure on $Y^{3}$. Clearly, the polarized $T$-structure on $M^{3}$ is not semi-injective.

Assume that $\Sigma^{2}$ has a positive genus. We claim that the universal covering of $M^{3}$ is not be contractible.

Let $C_{0}$, a circle, denote the boundary component of $\Sigma^{2}$ corresponding to the gluing. Take a simple path, $\alpha$, in $\Sigma^{2}$, whose end points are in $C_{0}$ and which goes around one of the holes of $\Sigma^{2}$. In this way, we obtain an embedded 2-sphere in $M^{3}$ which is formed by the cylinder, $\alpha \times S^{1}$, and the discs at its two boundary components. To see that this 2 -sphere does not bound a 3-ball in $M^{3}$, take a simple closed curve $\beta$ in $\Sigma^{2}$ which cuts transversally the hole which $\alpha$ encircles, and such that $\alpha$ and $\beta$ intersect at a single point. (For example, if $\Sigma^{2}$ has genus 1 , then one can think of $\alpha$, $\beta$ as independent generators of the fundamental group of the corresponding torus)

By the Seifert-van Kampen theorem, $\beta$ is not homotopically trivial in $M^{3}$. Since the intersection number of $\beta$ and our 2 -sphere is 1 , this 2 -sphere must be essential.

Assume that $\Sigma^{2}$ has genus zero, and (at least) 3 boundary components. In this case, we choose $\alpha$ a simple path with two ends in $C_{0}$, which is not homotopic to a segment in $C_{0}$ e.g., if $\Sigma^{2}$ looks like a pair of pants, then $\alpha$ cuts this pair of pants in half symmetrically. Let $\beta$ denote a simple closed curve in $M^{3}$ whose intersection with $\Sigma^{2}$ is a simple path connecting two other boundary components of $\Sigma^{2}$, such that $\alpha$ and $\beta$ intersect at a single point. As above, we obtain an essential 2-sphere in $M^{3}$.

Proof of Theorem 3.1.

Case 1. Assume that $\mathcal{F}$ is pure. If $\mathcal{F}$ is polarized, then $M^{3}$ is a Seifert fiber space whose base space is a surface of genus $\geq 1$ (otherwise $\widetilde{M}^{3}$ is not contractible) or a solvmanifold. In any case, $\mathcal{F}$ is pure and injective.

If $\mathcal{F}$ is not polarized, then each singular orbit is a circle. Since a tubular neighborhood of an isolated circle orbit is homeomorphic to a solid torus, one can replace the $T^{2}$-structure on the solid torus with a free $S^{1}$-action to obtain a mixed polarized $F$-structure; see Case 2. 
Case 2. Assume that $\mathcal{F}$ is a mixed polarized $F$-structure. Following [Ro], one sees that $M^{3}$ is obtained as by gluing together a finite number pieces, $\left\{\Sigma_{i}^{2} \times S^{1}\right\}$, each of which is a closed 3-manifold with boundary, where each $\Sigma_{i}^{2}$ is homeomorphic to one of the following types of surface with boundary:

I) $\Sigma_{i}^{2}$ is a disk $D^{2}$.

II) $\Sigma_{i}^{2}$ is a surface of genus zero with at least three boundary components. (In particular, $\Sigma_{i}^{2}$ is not a cylinder.)

III) $\Sigma_{i}^{2}$ is a surface of positive genus.

We say that $\Sigma_{i}^{2} \times S^{1}$ is of type I, II or III, if $\Sigma_{i}^{2}$ is of type I, II or III, respectively. We claim the following:

(3.3.1) Gluing any type I piece to a type II or III cannot kill the homotopy class of the $S^{1}$-factor of the type II or III

First assuming (3.3.1), we prove the existence of the injective $F$ structure. We observe:

(3.3.2) Any boundary component of a type II or a type III piece is incompressible i.e. the inclusion induces an injection on the fundamental groups.

(3.3.3) If a type I piece is glued to a type III, as in (3.3.1), then the remaining boundary components (if such exist) stay incompressible.

(3.3.4) If, whenever possible, a type I piece is glued to a type II as in (3.3.1), then either the result homeomorphic to a solid torus, or the boundary is disconnected and incompressible.

Relation (3.3.4) implies that $M^{3}$ cannot be obtained just by gluing type I pieces to a single type II. In this case, $M^{3}$ is homeomorphic to a lens space or to $S^{2} \times S^{1}$, which is not possible; for details, see Section 4 of [Ro].

Let $\left\{X_{i}\right\}$ denote the manifold obtained by gluing type I pieces to type II's whenever possible. By (3.3.4), we can assume that each $X_{i}$ has more than one incompressible boundary component.

Similarly, let $\left\{Y_{j}\right\}$ denote the result of gluing type I pieces to type III's whenever possible. By (3.3.3), the boundary of $Y_{j}$ is incompressible. By (3.3.1), each $X_{i}$ (respectively $Y_{j}$ ) admits an $S^{1}$-action which is the extension of the $S^{1}$ rotation on the type II (respectively III) piece. By Van Kampen's theorem, the incompressibility of the boundaries implies that the $F$-structure generated by these $S^{1}$-actions on $X_{i}, Y_{j}$ is injective. 
We now verify (3.3.1). We show that if (3.3.1) is false, then there is an essential sphere in $M^{3}$. Thus, the universal covering of $M^{3}$ is not contractible; a contradiction.

If some gluing of a type I piece to a type III kills the homotopy class of the $S^{1}$-factor, then we are in the situation discussed in the case of Example 3.2 in which $\Sigma^{2}$ has positive genus. As in that case, we conclude that there exists an essential 2-sphere in $M^{3}$.

If some gluing of a type I piece to a type III kills the homotopy class of the $S^{1}$-factor, then by (3.3.4) we are in the case of Example 3.2 in which $\Sigma^{2}$ has genus zero. As in that case, we conclude the existence of an essential 2-sphere in $M^{3}$.

\section{Semirigidity.}

\section{a. Semirigidity versus rigidity.}

In this section we try to put Theorem $\mathrm{C}$ in a more general context.

By the moduli space of metrics of a given type on a (pointed) closed manifold, we mean the collection of isometry classes of such metrics, equipped with the topology of (pointed) Gromov-Hausdorff convergence. Let $\mathcal{G}_{<0}^{*}\left(M^{n}\right)$, the moduli space of complete metrics of nonpositive curvature, denote the subset of the full moduli space consisting of those metrics with nonpositive curvature.

We say that $\mathcal{G}_{<0}^{*}\left(M^{n}\right)$ is rigid if up to rescaling, any two metrics in $\mathcal{G}_{\leq 0}^{*}\left(M^{n}\right)$ are isometric. The manifolds to which classical rigidity theorems apply all have the property that every finite Riemannian covering of $M^{n}$ is irreducible (i.e. not a metric product) and the Riemannian universal covering is not a metric product with nontrivial flat factor.

Mostow's rigidity theorem states that the subset of $\mathcal{G}_{\leq 0}^{*}\left(M^{n}\right)$ consisting of locally symmetric metrics is rigid.

The rank of a maximal geodesic $\gamma$ is the dimension of the space of parallel Jacobi fields along $\gamma$. The rank of $M^{n}$ is the infimum of the rank of all maximal geodesics. If $M^{n}$ is also simply connected, then $M^{n}$ has rank $k$ means that at each point and in every direction, there is a $k$-flat i.e. a totally geodesic flat $k$-plane.

According to the higher rank rigidity of Ballmann, Burns-Spatzier, $g \in \mathcal{G}_{\leq 0}^{*}\left(M^{n}\right)$ is locally symmetric if $g$ has rank at least 2 ; see [Ba], [BS]. Moreover, Gromov showed that $\mathcal{G}_{<0}^{*}(M)$ is rigid if $\mathcal{G}_{<0}^{*}\left(M^{n}\right)$ contains a locally symmetric metric of rank at least two; see [BGS]. A typical example to

which these results apply is that of irreducible compact quotient manifold 
of $\widetilde{M}^{n}=S L(n, \mathbb{R}) / S O(n, \mathbb{R})$ which has rank $\geq 2$.

In the present paper, we have studied certain (partial) rigidity phenomena for a class of nonpositively manifolds which does not intersect the one to which the rigidity theorem apply. These manifolds have the property that a certain aspect of their topology is visible in the local splitting structure of every nonpositively curved metric. Our most general such result is Theorem A, while the one with the strongest conclusion is Theorem $\mathrm{C}$ which concerns the 3-dimensional case. Conjecturally the conclusion of Theorem $\mathrm{C}$ holds for a natural class of higher dimensional manifolds as well; see Conjecture 4.2. To this end, we repeat here the definition given in Section 1.

Definition 4.1. The space, $\mathcal{G}_{\leq 0}\left(M^{n}\right)$, is semirigid, if there exists a $C r$ structure, $\mathcal{C}_{r}$, on $M^{n}$, such that every $g \in \mathcal{G}_{\leq 0}\left({ }^{n} M\right)$ has a local splitting structure compatible with $\mathcal{C}_{r}$.

\section{b. Semirigidity conjectures.}

Gromov defined the minimal volume, $\operatorname{MinVol}\left(M^{n}\right)$, to be the infimum of the volumes for all complete metrics on $M^{n}$ with normalized curvature $|\sec | \leq 1$. Since a $C r$-structure is in particular, an atlas for a polarized $F$-structure, semirigidity of $\mathcal{G}_{\leq 0}\left(M^{n}\right)$ implies $\operatorname{Min} \operatorname{Vol}\left(M^{n}\right)=0$.

Conjecture 4.2. If $X^{n}$ admits an F-structure and $f: X^{n} \rightarrow M^{n}$ is a homotopy equivalence, then every metric of nonpositive sectional curvature on $M^{n}$ admits an abelian splitting structure and hence, a compatible $\mathrm{Cr}$ structure.

Following [GW], [LY], [CG1], it is not hard to see that Conjecture 0.3 holds if $X^{n}$ admits a pure F-structure; see Corollary 4.5.

In the following conjecture, we strengthen both the hypothesis and conclusion of Conjecture 4.2 .

Conjecture 4.3. If $M^{n}$ is closed, then $\mathcal{G}_{\leq 0}\left(M^{n}\right)$ is semirigid if and only if $\operatorname{Min} \operatorname{Vol}\left(M^{n}\right)=0$.

\section{c. Minimal $d$-volume and pure structures.}

Given $d>0$, the $d$-minimal volume $\operatorname{MinVol}_{d}\left(M^{n}\right)$ is the infimum of volumes of all complete metrics on $M^{n}$ with $|\sec | \leq 1$ and diam $\leq d$. According to $[\mathrm{CR}]$, there is a constant $\epsilon(n, d)>0$ such that if $\operatorname{MinVol}_{d}\left(M^{n}\right)<\epsilon(n, d)$, then $\operatorname{Min} \operatorname{Vol}\left(M^{n}\right)=0$. 
For manifolds whose $d$-minimal volume is sufficiently small, the semirigidity conjecture holds.

Theorem 4.4. There is a constant, $\epsilon(n, d)>0$, such that if a closed manifold, $M^{n}$, satisfies $\operatorname{Min}_{\operatorname{Vol}}\left(M^{n}\right)<\epsilon(n, d)$, then $\mathcal{G}_{\leq 0}\left(M^{n}\right)$ is semirigid.

Proof. By [CG2], $M^{n}$ admits an $F$-structure, $\mathcal{F}$, whose orbits have positive dimension. Since the volume is small relative to the diameter, $\mathcal{F}$ is pure; see [Fu1], [Fu2], [CFG].

By [CG1] (see the appendix) any pure $F$-structure on a closed manifold whose universal covering is contractible must be injective. Hence, $\mathcal{F}$ is injective.

Recall that an orbit is regular if it has a neighborhood such that the orbits form a fibration. Let $\mathcal{B}$ denote the subgroup of $\pi_{1}\left(M^{n}\right)$ generated by loops in a regular orbit. The fact that $\mathcal{F}$ is injective, implies that $\mathcal{B}$ is Bieberbach of positive rank. Since regular orbits form a connected open dense subset, it follows that $\mathcal{B}$ is a normal subgroup of $\pi_{1}\left(M^{n}\right)$.

By $[\mathrm{GW}],[\mathrm{LY}]$, for any $g \in \mathcal{G}_{\leq 0}\left(M^{n}\right)$, the riemannian universal covering space, $\widetilde{M}^{n}$ splits,

$$
\widetilde{M}^{n}=\operatorname{Min}(\mathcal{B})=\widetilde{M}_{0}^{n-k} \times \mathbb{R}^{k},
$$

where $k=\operatorname{rank}(\mathcal{B})$. By Proposition 5.11 [CCR], a finite covering space of $M^{n}$ is diffeomorphic to $M_{0}^{n-k} \times T^{k}$. Note that the single chart, $\left(M_{0}^{n-k} \times\right.$ $\left.T^{k}, M^{n}, T^{k}\right)$, defines a $C r$-structure which is compatible with the abelian local splitting structure of $g$. Since the abelian local splitting structure is determined by $\mathcal{B}$, the $C r$-structure is also compatible with the abelian local splitting structure of any metric in $\mathcal{G}_{\leq 0}\left(M^{n}\right)$.

The proof of Theorem 4.4 also yields the following:

Corollary 4.5. Let $M^{n}$ be a closed manifold admitting at least one metric of nonpositive curvature. If $M^{n}$ carries a pure nontrivial $F$-structure, then $\mathcal{G}_{\leq 0}\left(M^{n}\right)$ is semirigid.

\section{References.}

[Ba] W. Ballmann, Nonpositively curved manifolds of higher rank Ann. of Math 122 (1985), 597-609.

[BGS] W. Ballman, M. Gromov, and V. Schroeder, Manifolds of nonpositive curvature, Basel: Birkhäuser, Boston, Basel Stuttgart, (1985) 
[BS] K. Burns and R. Spatzier, Manifolds of nonpositive curvature and their buildings, Math. IHES 65 (1987), 137-145.

[Bu1] S. Buyalo, Volume and the fundamental group of a manifold of nonpositive curvature, Math. USSR Sbornik 50 (1985), 137-150.

[Bu2] S. Buyalo, Collapsing manifolds of nonpositive curvature I, Leningrad Math. J. vol. 1 No. 5 (1990), 1135-1155.

[Bu3] S. Buyalo, Collapsing manifolds of nonpositive curvature II, Leningrad Math. J. vol. 1 No. 6 (1990), 1371-1399.

[Bu4] S. Buyalo, Homotopy invariance of some geometric properties of nonpositive curved three-manifolds, St. Petersburg Math. J. 3 (1992), 791-808.

[Bu5] S. Buyalo, Three-dimensional manifolds with $C r$-structures, Some Questions of Geometry in the Large, A.M.S. Translations 176 (1996), $1-26$.

[CCR] J. Cao, J. Cheeger, and X. Rong, Splittings and $C r$-structures for manifolds with nonpositive sectional curvature, Invent. Math. 144 (2001), 139-167.

[CFG] J. Cheeger, K. Fukaya, and M. Gromov, Nilpotent structures and invariant metrics on collapsed manifolds, J. A.M.S., 5 (1992), 327372 .

[CG1] J. Cheeger and M. Gromov, Collapsing Riemannian manifolds while keeping their curvature bounded I, J. Diff. Geom. 23 (1986), 309-364.

[CG2] J. Cheeger and M. Gromov, Collapsing Riemannian manifolds while keeping their curvature bounded II, J. Diff. Geom. 32 (1990), 269298.

[CR] J. Cheeger and X. Rong, Existence of polarized $F$-structures on collapsed manifolds with bounded diameter and curvature, GAFA, Geom. Funct. Anal. 5 (1996), 411-429.

[ES] J. Eells, and J. H. Sampson, Harmonic mapping of Riemannian manifolds, Amer. J. Math. 86 (1964), 109-160.

[Fu1] K. Fukaya, Collapsing Riemannian manifolds to ones of lower dimension, I, J. Diff. Geom. 25 (1987), 139-156. 
[Fu2] K. Fukaya, Collapsing Riemannian manifolds to ones of lower dimension, II, J. Math. Soc. Japan 41 (1989), 333-356.

[GW] D. Gromoll and J. Wolf, Some relations between the metric structure and the algebraic structure of the fundamental group in manifolds of nonpositive curvature, Bull. Am. Math. Soc. 77, No. 4 (1971), $545-552$.

[Gr] M. Gromov, Volume and bounded cohomology, Publ. Math., IHES 56 (1982).

[Har] P. Hartman, On homotopic harmonic maps, Canada, J. Math. 19 (1967), 673-687.

[LY] B. Lawson and S.T. Yau, On compact manifolds of nonpositive curvature, J. Diff. Geom. 7 (1972).

[Le] B. Leeb, 3-manifolds with(out) metrics of nonpositive curvature, Invent. Math. 122 (1995), 277-289.

[Pr] A. Preissmann, Quelques propriétés des spaces de Riemann, Comment. Math. Helv 15 (1942), 175-216.

[Ro] X. Rong, Limiting eta-invariants of collapsed three-manifolds, J. Diff. Geom. 37 (1993), 535-568.

[Sc] V. Schroeder, Rigidity of nonpositively curved graph manifolds, Math. Ann. 274 (1986).

[Wa1] F. Waldhausen, Eine klasse von 3-dimensionalen mannigfalkeitigen, I, Invent. Math. 3 (1967), 308-333.

[Wa2] F. Waldhausen, Eine klasse von 3-dimensionalen mannigfalkeitigen, II, Invent. Math. 4 (1967), 87-177.

JiAnguO CAO

Mathematics Department

UNIVERSITY OF Notre DAME

Notre DAMe, IN 46556

JefF Cheeger

Courant Institute of Mathematical Sciences

NEW YoRK, NY 10012 
XiaOchun Rong

Mathematics Department

RUTGERS UNIVERSITY

New Brunswick, NJ 08903

and

Mathematics Department

Capital Normal University

BeiJing, P.R.C. 